\title{
MONITORAMENTO DE EQUIPAMENTOS DAS PONTES ROLANTES NA ARCELORMITTAL VEGA*
}

André Santos Caires de Sousar ${ }^{1}$ Northon Torezani Cavazzoni

\section{Resumo}

As equipes de manutenção das pontes de Vega não possuíam uma ferramenta de armazenamento de dados de longo prazo, que permitisse analise de falhas e a avaliação das condições operacionais necessárias à manutenção preditiva. Com essa deficiência, as condições de ocorrência de paradas não programadas eram favorecidas. Fazia-se necessário uma ferramenta que permitisse o desenvolvimento de aplicações mais sofisticadas para monitoramento de equipamentos das Pontes Rolantes, além de que todo diagnóstico realizado era localmente na ponte e com as informações atuais (posteriores ao evento ocorrido). Após a implantação os ganhos foram satisfatórios, reduziu-se o tempo de atendimento das ocorrências, a planta se adequou ao padrão mundial de Prevenção de Fatalidades do grupo ArcelorMittal, reduzindo o trabalho em altura, exposição do executante ao risco, custo de manutenção e quantidade de paradas não planejadas, além de suportar o foco na manutenção preventiva e preditiva.

Palavras-chave: PIMS; Preditiva, Ponte Rolante, Prevenção de acidentes.

\begin{abstract}
MONITORING OF CRANE EQUIPMENT AT ARCELORMITTAL VEGA
Abstract

Vega Crane maintenance teams didn't have a long-term data storage tool that would allow failure analysis and evaluation of the operational conditions required for predictive maintenance. With this deficiency, the conditions for occurrence of unscheduled stops were favored. A tool was necessary to allow the development of more sophisticated applications for monitoring of the equipment of the Cranes, besides that all diagnosis was made locally in the Crane and with the present Information (subsequent to the event that occurred). After a successful implementation, the time taken to deal with the occurrences was reduced, a plant complies with the ArcelorMittal Group's worldwide Fatality Prevention standard, reducing work height, risk exposure, maintenance cost and quantity unplanned stops, as well as supporting the focus on preventive and predictive maintenance.
\end{abstract}

Keywords: PIMS; Predictive, Crane, Accident Prevention.

1 Engenheiro da Computação, Especialista em Desenvolvimento de Automação, ArcelorMittal Vega, São Francisco do Sul, SC, Brasil.

2 Engenheiro Eletricista, Especialista em Desenvolvimento de Automação, ArcelorMittal Vega, São Francisco do Sul, SC, Brasil. 


\section{INTRODUÇÃO}

As equipes de manutenção das pontes de Vega careciam de uma ferramenta, que as auxiliassem nas analise de falhas e na avaliação das condições operacionais necessárias à manutenção preditiva. Devido a essa deficiência, as condições de ocorrência de paradas não programadas eram favorecidas, foi apresentado o PIMS como solução.

PIMS $^{1}$ são sistemas que coletam dados de processo de diversas fontes, os armazenam num banco de dados histórico e os disponibilizam através de diversas formas de apresentação. O PIMS na ArcelorMittal - Vega foi implementado diante da necessidade de resolver o problema da fragmentação de dados e proporcionar uma visão unificada do processo. Inicialmente, o PIMS passa a se constituir na ferramenta fundamental para o engenheiro de processos e manutenção. A partir de uma estação, o usuário pode visualizar tanto os dados de tempo real como históricos da planta, montar tabelas, gráficos de tendência e sinópticos, concentrando em uma única base de dados todas as informações sobre os aspectos de um equipamento especifico.

Através de rotinas cíclicas, o banco de dados temporal é alimentado como os dados de processo da planta, com as quais o usuário pode visualizar através de tendências ou outras aplicações (telas sinópticas, planilhas em Excel, notificações por e-mail), vide figura 1.

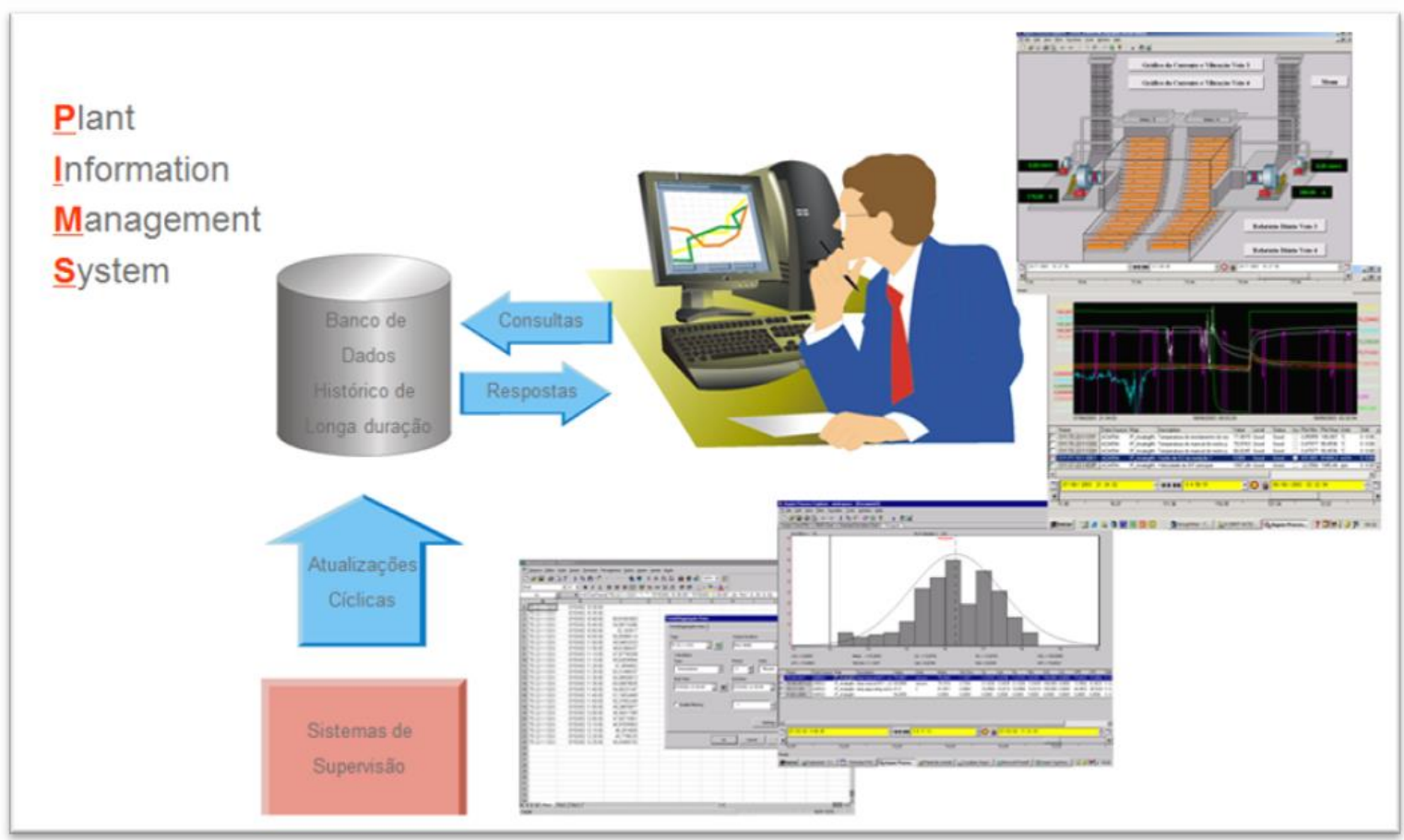

Figura 1 - Diagrama simplificado do Sistema PIMS em Vega

\footnotetext{
${ }^{1}$ Plant Information Management System
} 


\section{MATERIAIS E MÉTODOS}

O projeto se deu inicio com a necessidade de ter um sistema que possuísse informação confiável e centralizada, para suprir a necessidade de monitoramento e gestão dos indicadores das Pontes. Aos envolvidos foi apresentando o potencial da ferramenta a qual possui uma grande capacidade para geração de valor.

Com o fato do tempo necessário para fazer atendimentos nas pontes, inspeções de rotina, além dos procedimentos de segurança para acessar as mesmas e realizar trabalhos em altura, ser relativamente alto, a equipe responsável pelas pontes iniciou um estudo para reduzir estes tempo. A figura 2 exibe uma atividade rotineira da manutenção.

Na figura 2 é demonstrado a altura de exposição do executante esta exposto ao risco, bem como a dificuldade de acesso e a ergonomia na atividade envolvimento acesso à lógica de programação da ponte.

Em média, cerca de 1 hora por dia era gasta para realizar inspeções de rotina nas pontes. É importante mencionar que para a realização de trabalhos em altura, o empregado precisa realizar os testes de pressão arterial e alcoolemia (cerca de 20 minutos), além dos preparativos com os equipamentos de segurança.
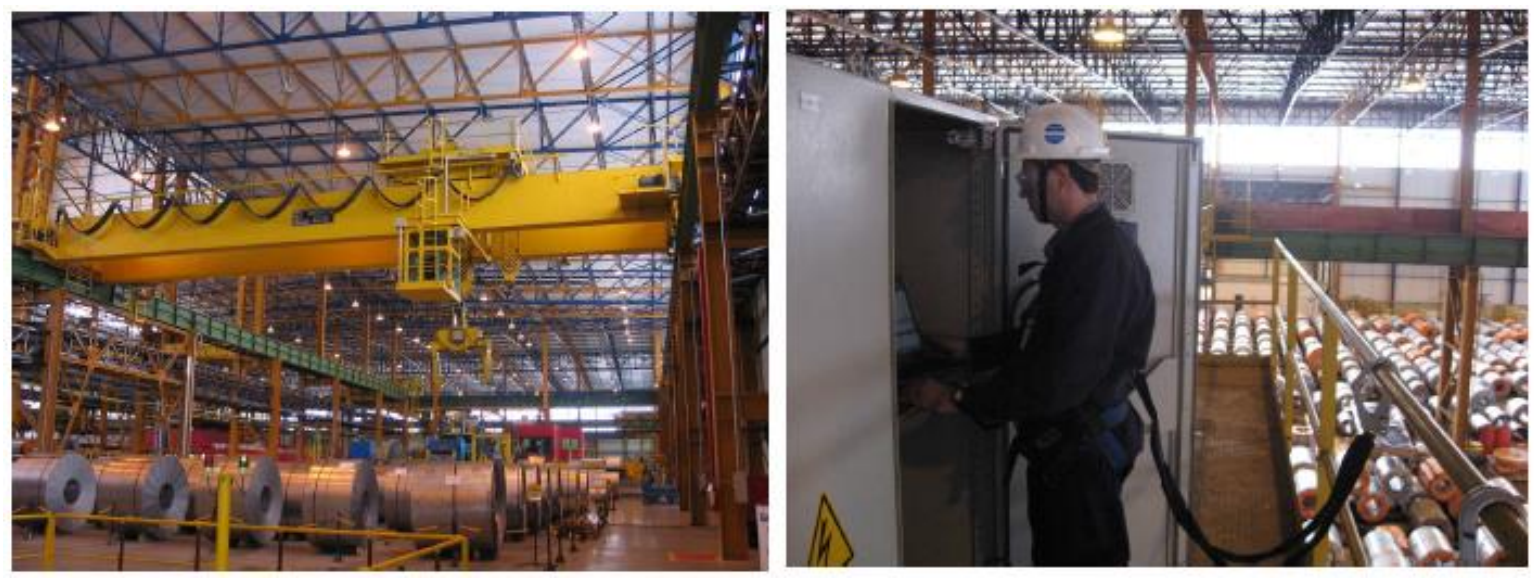

Figura 2 - Atividade de manutenção na Ponte Rolante

As verificações de permissíveis na lógica e/ou falhas precisavam ser realizadas localmente no CLP2 da ponte, pois o mesmo não possuía outro tipo de comunicação a não ser a serial, a qual era conectada diretamente em um notebook.

De inicio, foi optada a instalação de estações com supervisório nas áreas. O objetivo era permitir um diagnóstico prévio das falhas e realizar um monitoramento online.

Junto com o início desta iniciativa, o projeto PIMS se encaminhava para a fase de criação das aplicações e propagação de sua utilização nas áreas. Devido à proximidade das áreas, a qual possibilitou uma grande sinergia entre as equipes, a

\footnotetext{
${ }^{2}$ Controlador Lógico Programável
} 
equipe do projeto PIMS viu na iniciativa das pontes, a oportunidade de ganho utilizando este sistema em contrapartida com apenas o uso do sistema supervisório.

A proposta com o PIMS ainda em sua ideia inicial, já apresentava economias e ganhos em relação à utilização do supervisório. Entre eles, já se destacavam a não necessidade de gastos com novas estações e licenças de supervisório, cabeamento de rede e energia para as novas estações, bem como ter os valores históricos das variáveis dos controladores.

Como estratégia do projeto, primeiramente foi realizado o envolvimento dos profissionais da área da manutenção e operação. Para estes, de início, foi apresentando o potencial da ferramenta, a qual possui uma grande capacidade para geração de valor.

Adotando a estratégia de mesclar a experiência dos profissionais das pontes com o conhecimento da aplicabilidade da ferramenta, foi possível a criação de várias ideias, bem como, a sugestão de melhorias nas pontes para o uso futuro de outras utilidades da ferramenta.

Desta forma, foram iniciadas as principais fases do projeto, sendo elas:

- Avaliação de requisito

- Mapeamento das aplicações por ponte.

A primeira etapa da análise foi identificar a maior quantidade possível de ideias e necessidades, foi agendada reuniões com os Especialistas da manutenção e os Técnicos, utilizando a abordagem de brainstorming, para identificar as oportunidades em potencial, dificuldades e iniciativas que interagem com o equipamento.

Por instigar a aplicação de técnicas oferecidas pela ferramenta, adicionando a estas a experiência e conhecimento empírico da manutenção e processo. Com isso, um ponto muito citado foi a geração de aplicações baseada em desempenho.

Após a definição dos requisitos do projeto, foi definida a estrutura física e lógica da comunicação entre os controladores e o PIMS. Na figura 3 é demonstrado um diagrama de comunicação simplificado. 


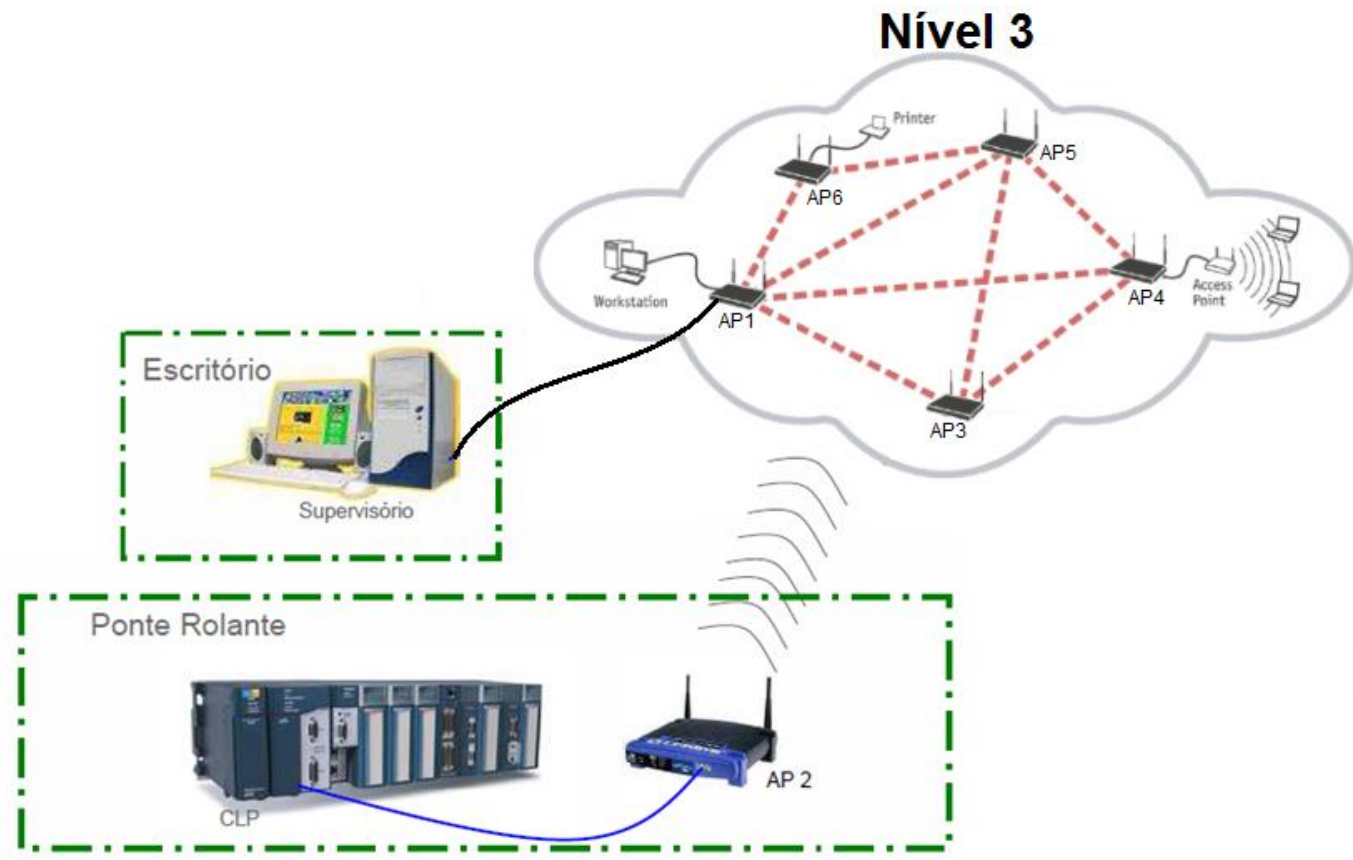

Figura 3 - Diagrama simplificado da comunicação

A segunda etapa foi a estruturação que envolveu o agrupamento dos itens relacionados, segundo uma classificação de prioridade ordenada pelo menor esforço de execução e maior retorno.

Embora as Pontes Rolantes em Vega já possuíssem CLP, foi necessário realizar a troca de algumas CPU's, para que as mesmas suportassem conexão Ethernet.

Os pátios, já havia cobertura de rede Wireless industrial e foi necessário um estudo na estrutura de rede para criação regras de acesso, para permitir o tráfego entre a rede de Nível 3 e a rede de produção Nível 1. Em cada ponte rolante foi instalado um Access Point cliente (AP2), o qual realiza roaming entre os Access Points instalados nos vãos (representados na figura 3 como AP1).

Outra fase importante do projeto foi à especificação das aplicações para desenvolvimento, embora já tivéssemos mapeado os itens mais relevantes, o desafio foi transformar os itens anteriormente baseados na manutenção temporal para uma manutenção baseada em desempenho dos equipamentos e processos associados. Além disso, a ferramenta iria suportar a gestão das pontes através de indicadores operacionais.

Um aspecto importante no projeto foi relativo à revisão do procedimento de segurança, relativo aos atendimentos de manutenção na ponte, em caso de uma intervenção no programa do CLP, somente após a liberação do operador da ponte rolante e com a confirmação de que a mesma esteja totalmente parada, será permitida a alteração na lógica do controlador, afim, de garantir a segurança do operador e de outros colaboradores que estejam dividindo o mesmo ambiente de trabalho com ponte rolante, evitando acidentes. 


\section{RESULTADOS OBTIDOS}

Ao final do projeto os ganhos foram satisfatórios, pois anteriormente o funcionário precisava acessar a ponte para identificar qual permissível impedia o funcionamento da mesma, e não havia registro de histórico para consultas ou análise de falhas, conforme a aplicação mostrada na figura 4. Depois da implantação, o profissional da manutenção possui acesso a toda a lógica de permissíveis da ponte de forma segura e explicita, sendo mais ágil na atuação necessária.

Nos ganhos deste projeto, destacam-se a economia de $\mathrm{R} \$ 13.000,00$ com licenciamento de sistema supervisório, a redução de custo de manutenção, o aumento da produtividade da equipe técnica, economia de $R \$ 30.000,00$ devido a não aquisição de novas estações e instalação de infraestrutura. $O$ desenvolvimento de aplicações de supervisórios foi substituído pelas aplicações no PIMS, gerando uma economia de $\mathrm{R} \$ 150.000,00$.

Em uma estimativa com a equipe de manutenção foi calculado uma redução de $22 \mathrm{~h} / \mathrm{mês}$ nas atividades de inspeção de cada ponte.

Além dos ganhos tangíveis, o projeto resultou em mais segurança, pois não é mais necessário acessar a ponte para realizar o diagnostico prévio, ganhando tempo na tomada de decisões.

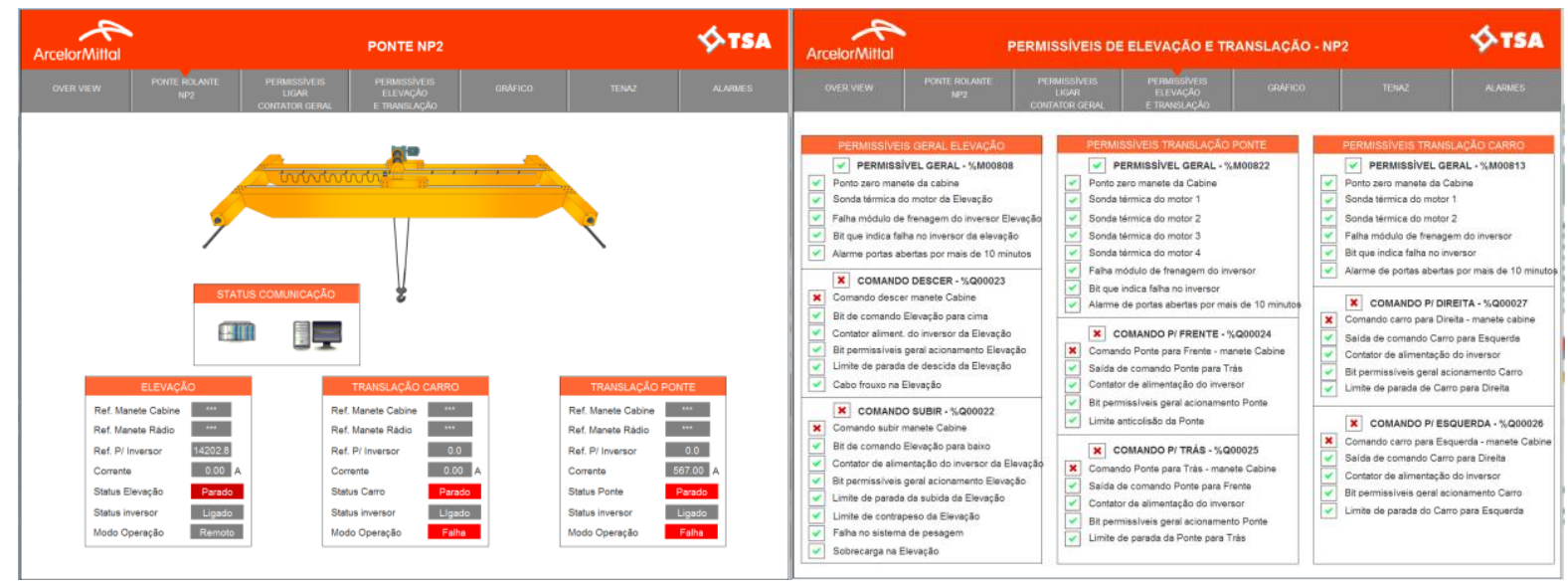

Figura 4 - Aplicações de diagnostico.

Com o objetivo de auxiliar as equipes de Processo, Planejamento e Programação, foi criado e disponibilizado em tempo real, o status de cada linha e o tempo de parada das mesmas, conforme demonstrado na Figura 5. De acordo com os critérios estabelecidos pela equipe de Planejamento e Programação, foram criados alertas de notificação por e-mail, para eventos de inicio e fim de paradas dos equipamentos. Estas implementações já estão ajudando a equipe de Programação a atuar na estratégia de produção. 

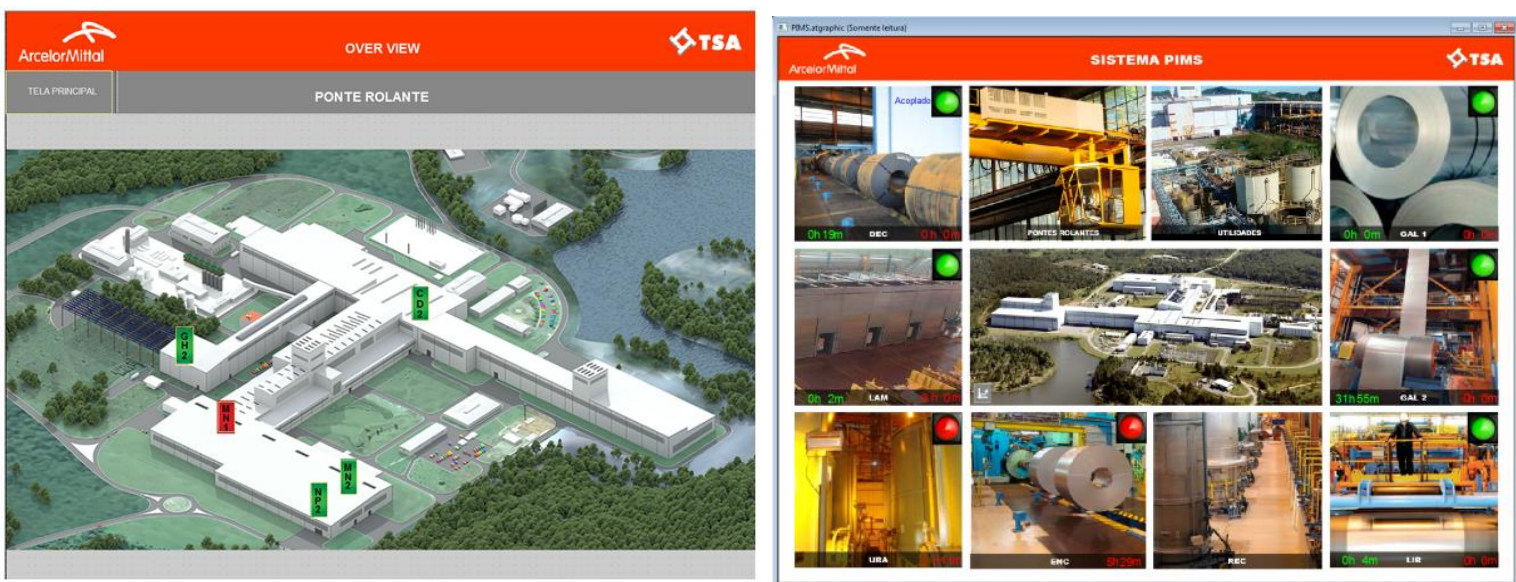

Figura 5 - Aplicações de Visão Geral de Processo e Ponte Rolante.

Foram definidos indicadores de tempo de funcionamento das pontes, para avaliar e otimizar a mão de obra operacional das mesmas e a gestão de manutenção. $\mathrm{Na}$ Figura 6, os indicadores mostram o tempo de ociosidade de uma ponte específica junto à algumas informações gerais da ponte.
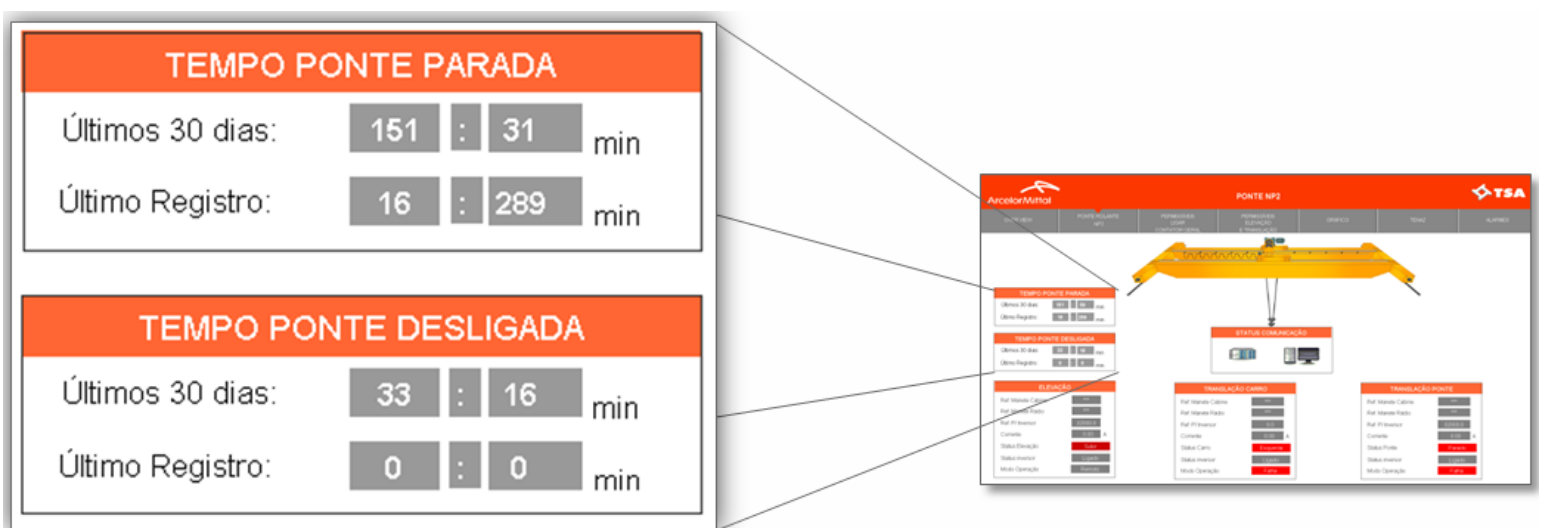

Figura 6 - Aplicações de Indicadores.

Criação de indicador de distância percorrida (Figura 7), possibilitando a manutenção de equipamentos pelo uso e não pela data da última atuação. Um exemplo a ser citado é a possibilidade da troca de rodas pela distância e não por tempo. Com esta iniciativa, a gestão do estoque de rodas e equipamentos dependentes do uso ficou mais precisa, mudando a forma de manutenção de manutenção dos equipamentos de temporal para condicional. Outros exemplos a serem citados são as verificações da contagem dos encoders para identificação de desvios, contagem de abertura e fechamento da tenaz e o acompanhamento das temperaturas dos inversores e motores. 


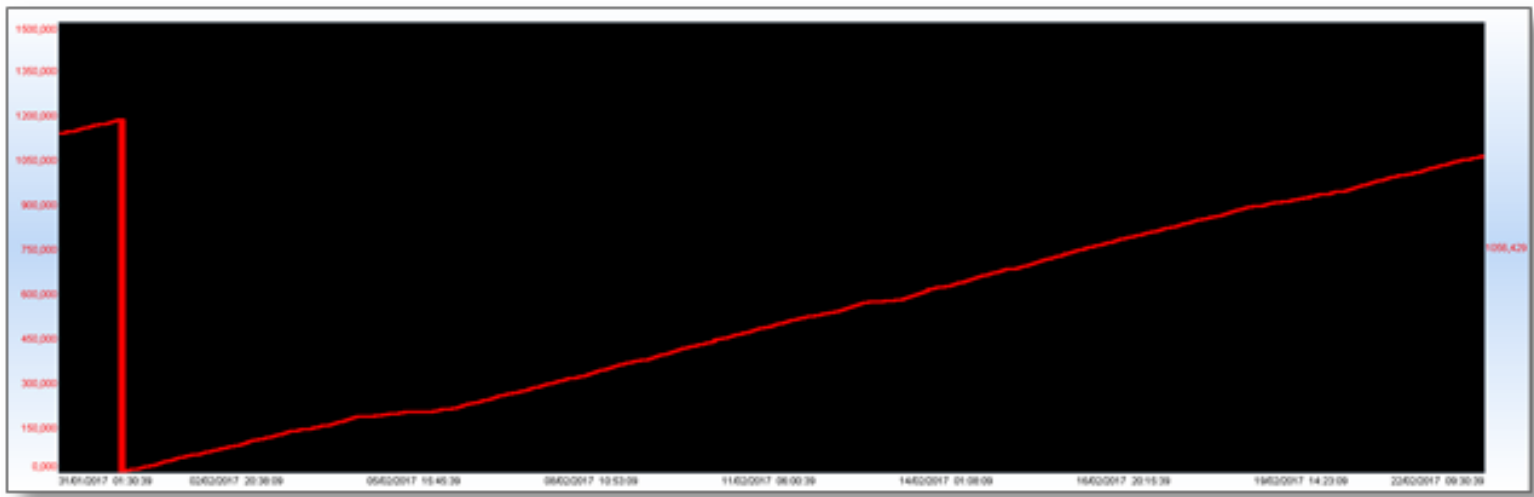

Figura 7 - Gráfico de distancia percorrida pela ponte (Troca das Rodas).

Criação de uma visão geral dos bloqueios (Figura 8) de movimentação, possibilitando um prévio diagnóstico com mais agilidade. Com essa aplicação o usuário consegue identificar intuitivamente o motivo de a lógica estar impedindo a movimentação do equipamento da ponte.

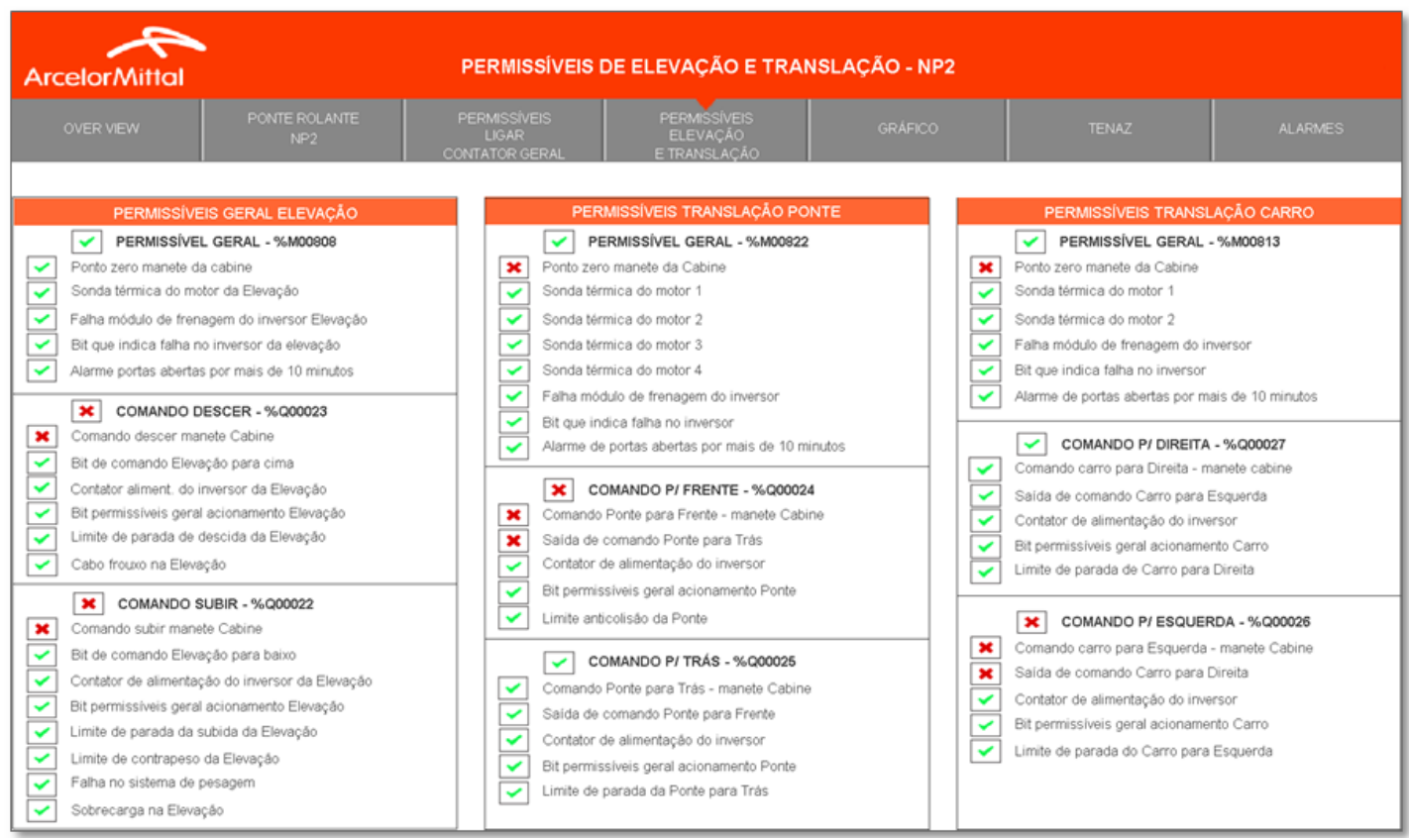

Figura 8 - Aplicações da Ponte Rolante. 


\section{CONCLUSÃO}

Ao final do projeto tivemos o envolvimento das equipes de manutenção, operação, controle de processo e confiabilidade. Com relação ao feedback dos envolvidos, as aplicações sinópticas, relatórios e alarmes desenvolvidas agregaram valor para os Padrões Mundiais de Prevenção de Fatalidades do grupo ArcelorMittal, referente ao trabalho em altura, reduzindo exposição do executante ao risco.

Com a maturidade no uso da ferramenta, os próprios usuários serão capazes de realizar as analises necessárias e identificar as particularidades de cada ponte e/ou suas características comuns. De posse destas informações será possível ponderar a influência de cada uma destas características na performance dos equipamentos;

O próximo passo do projeto será concluir a implementação nas demais pontes e desenvolver aplicações que irão interagir com o atual sistema de manutenção (ARMP), gerando ordens de serviço de forma automática para execução ou inspeção de serviços em pontes rolante.

Com a experiência do projeto, ficou uma rica coleção de lições aprendidas, que certamente irão contribuir muito para o sucesso dos próximos, bem como 0 aperfeiçoamento dos processos de gestão, com um consequente aculturamento das equipes envolvidas.

\section{REFERÊNCIAS}

1 SOUSA, A.S.C. Especialista Desenvolvimento Automação da ARCELORMITTAL VEGA, 2017, São Francisco do Sul - SC.

2 CAVAZZONI,N.T. Especialista Desenvolvimento Automação da ARCELORMITTAL VEGA, 2017, São Francisco do Sul - SC. 